\title{
Síndrome hepatorrenal: Revisión de la literatura
}

\author{
Ignacio Villanueva Bendek ${ }^{1}$ \\ 1 Endomedica Ltda. Unidad Renal Davita, Bogotá, Colombia
}

\begin{abstract}
Resumen
El síndrome hepatorrenal (SHR) es una complicación frecuente y severa en pacientes con cirrosis hepática e hipertensión portal y se caracteriza por anormalidades circulatorias que llevan a vasoconstricción renal, generando insuficiencia renal funcional.

$\mathrm{Su}$ fisiopatología ha sido estudiada y el pronóstico es reservado a menos que el paciente reciba trasplante hepático.

El tratamiento medicamentoso con vasoconstrictores esplénicos es la esperanza futura de estos pacientes mientras acceden a trasplante hepático.
\end{abstract}

Palabras clave: Síndrome hepatorrenal, Insuficiencia renal, Cirrosis hepática, Tratamiento.

\section{Hepatorenal syndrome: up todate}

\begin{abstract}
The hepatorenal syndrome is a frequent and severe complication in patients with severe liver disease and portal hypertension, characterized by circulatory abnormalities that lead to renal vasoconstriction, generating a functional renal failure. Its pathophysiology to date is studied and unfortunately his prognosis is ominous, unless the patient is a candidate for liver transplantation (LT). Drug therapy with vasoconstrictors splenic vasculature is today a beacon of hope to reverse in a physiological way to bridge this syndrome and transplantation therapy as definitive treatment is achieved.
\end{abstract}

Keywords: Hepatorenal syndrome, Renal failure, Hepatic cirrhosis, Treatment

\section{Introducción}

E 1 síndrome hepatorrenal (SHR) es una patología potencialmente reversible que ocurre en pacientes con enfermedad hepática crónica tipo cirrosis, como también en pacientes con falla hepática aguda. Es caracterizado por una intensa vasoconstricción renal que lleva a una disminución de la percusión renal y de la rata de filtración glomerular ${ }^{1}$. Los estudios de histología renal en estos pacientes son normales o demuestran mínimas anormalidades que no explican el deterioro de la función renal, debido a esto es considerado un tipo de insuficiencia renal "funcional" en los pacientes cirróticos y es la causa más común de azoemia en este grupo de pacientes ${ }^{2}$. 
La naturaleza funcional de este tipo de falla renal ha sido reforzada por la ausencia de alteraciones en la histología de los riñones comprometidos, por estudios que demostraban que los riñones de pacientes cirróticos con SHR recuperaban la función renal normal cuando eran trasplantados a pacientes con insuficiencia renal terminal, sin enfermedad hepática o que el SHR es reversible después del trasplante hepático y, finalmente, por la reversibilidad del SHR con el tratamiento farmacológico. Revisaremos la fisiopatología, diagnóstico, clínica y tratamiento de esta patología.

\section{Fisiopatología}

Por varios años el SHR, se consideró asociado a 2 principales problemas: una falla hepática terminal e irreversible y una falla renal funcional secundaria a vasoconstricción renal; la mayoría de investigadores consideraban que la relación entre los 2 tipos de falla: renal y hepática, era secundaria a un deterioro hemodinámico sistémico asociado a vasodilatación arterial en el árbol esplénico; sin embargo, durante la última década un cuerpo de evidencia científica sugiere un síndrome complejo que afecta mucho más que a solo 2 órganos, sugiriendo que el deterioro de la función circulatoria sistémica asociado al SHR no solamente afecta al riñón sino que compromete otras circulaciones regionales ${ }^{2}$.

Cuatro conceptos han emergido con el cúmulo de investigaciones ${ }^{3}$ :

1. La vasodilatación arterial extrarrenal ocurre, principalmente, a nivel del árbol esplénico, mientras que en otros árboles vasculares ocurre todo lo contrario, la vasoconstricción, como es en el caso del riñón, hígado y cerebro, lo que contribuirá al desarrollo de encefalopatía, fallas hepática y renal.

2. El gasto cardiaco en pacientes con SHR puede estar disminuido y ser insuficiente para responder a las necesidades del individuo.

3. La peritonitis bacteriana espontánea (PBE) es el evento precipitante más frecuente del SHR tipo 1.

4. El tratamiento farmacológico puede revertir el SHR y mejorar la supervivencia de este grupo de pacientes.
La vasodilatación del árbol esplénico es causada, principalmente, por la presencia del óxido nítrico y otras sustancias vasodilatadoras ${ }^{4}$. Temprano en el curso de la enfermedad, la disminución en la resistencia vascular sistémica es compensada por el desarrollo de circulación hiperdinámica (incremento de la frecuencia cardiaca y del gasto cardiaco), sin embargo, a medida que la enfermedad progresa y la vasodilatación arterial incrementa, la circulación hiperdinámica es insuficiente para corregir la hipovolemia arterial efectiva. La hipotensión arterial resultante conlleva a la activación de mecanismos compensadores: sistema renina angiotensina (SRA), sistema nervioso autónomo (SNA) y hormona antidiurética, llevando a una retención de agua, sodio $\mathrm{y}$, posteriormente, a la formación de ascitis e hiponatremia dilucional. En este estado avanzado de la enfermedad, el SRA y el SNA están marcadamente estimulados y la presión arterial es críticamente dependiente del efecto de estos sistemas sobre el tono vascular 5 .

Debido a que el árbol esplénico es resistente a la vasoconstricción de estos mecanismos compensadores (mediado por la angiotensina II, norepinefrina, vasopresina y endotelinas) por la liberación local de óxido nítrico y otras sustancias vasodilatadoras, el mantenimiento de la presión arterial se fundamenta en la vasoconstricción de territorios vasculares extra esplénicos como son el riñón y el cerebro. El SHR se desarrolla cuando hay un deterioro en el volumen arterial efectivo e hipotensión arterial severa ${ }^{6}$. La vasoconstricción intensa generada lleva a una marcada disminución de la perfusión renal, azoemia e incremento de la creatinina sérica (Crs). Por lo tanto, la vasoconstricción renal en el SHR es la consecuencia de un efecto simultáneo de numerosos mecanismos vasoactivos sobre la circulación intrarrenal $^{7}$. El segundo concepto es que la disminución del gasto cardiaco en los pacientes con SHR conlleva a la hipoperfusión renal. Los diferentes estudios realizados en pacientes con SHR con ascitis refractaria, demuestran que el gasto cardiaco está significativamente reducido comparado con los pacientes sin SHR (Figura 1).

El mecanismo del bajo gasto cardiaco en SHR es desconocido. Las anormalidades cardiacas se 


\section{Figura 1}

Mecanismos patogénicos del SHR

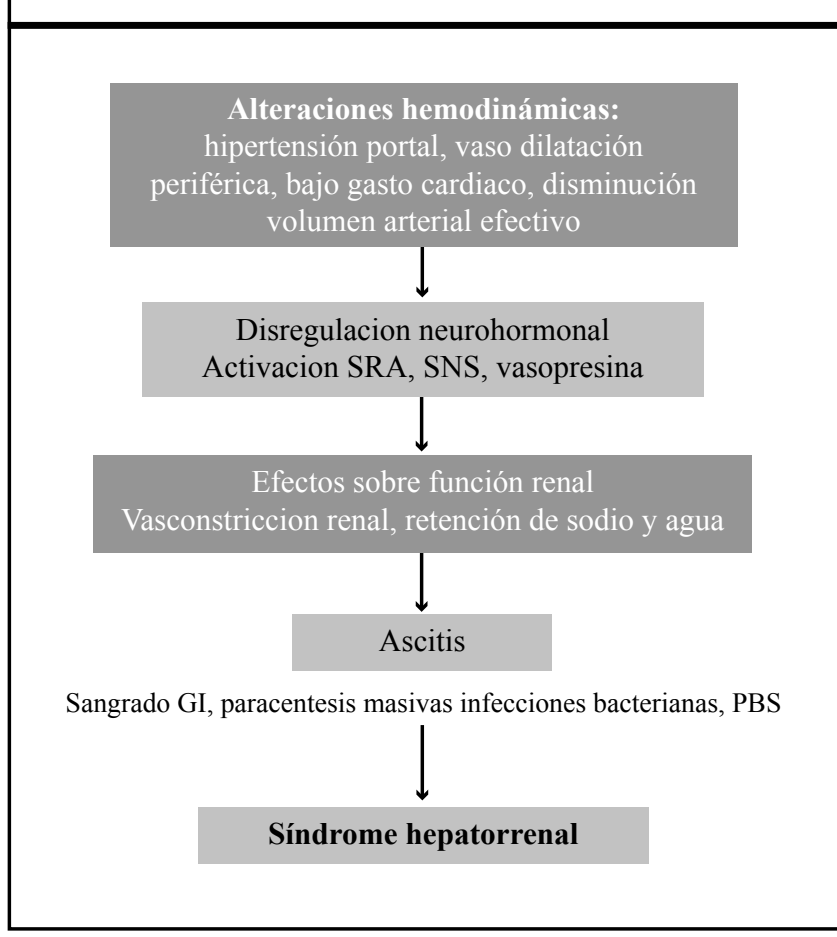

caracterizan por una atenuada respuesta sistólica y diastólica al estímulo, cambios en la repolarización e hipertrofia de las cavidades cardiacas, situación que se ha denominado como cardiomiopatía cirrótica. Una disminución de la precarga secundaria a una disminución del retorno venoso es una de las hipótesis.

El SHR puede desarrollarse en forma espontánea ${ }^{8}$, pero también ocurre por eventos precipitantes; los más frecuentes e importantes son infecciones como la peritonitis bacteriana espontánea, sangrado gastrointestinal y las paracentesis de grandes volúmenes sin la reposición adecuada de albúmina9

\section{Diagnóstico y aspectos epidemiológicos}

Hay que tener en cuenta que para cualquier definición de deterioro de la función renal o de injuria renal aguda en cualquier patología, incluyendo enfermedades hepáticas como la cirrosis o la insuficiencia hepática, la medición de la Crs sigue siendo el método más práctico y ampliamente aceptado para la estimación de la función renal en la práctica clínica, y es la base de las definiciones existentes de lesión renal aguda (AKI, por sus siglas en inglés). El impacto pronóstico de la función renal en la enfermedad hepática se refleja en la inclusión de la Crs en el modelo para la puntuación, en etapa final, de la enfermedad del hígado (MELD), que se utiliza para dar prioridad a los pacientes para el trasplante de hígado $^{10}$. Sin embargo, en la cirrosis, la Crs es notoriamente inexacta en el diagnóstico de la disfunción renal, ya que sobreestima la función renal debido a la disminución de la producción de creatinina por la malnutrición calórica hígado, proteína y pérdida de masa muscular ${ }^{10-13}$. Además, la medición de la creatinina sérica utilizando el método de Jaffe puede ocasionar disminuciones de los niveles de creatinina por la interferencia que causa la hiperbilirrubinemia $^{14}$ o por uso de cefalosporinas ${ }^{14,15}$.

La cistatina $\mathrm{C}$ se ha sugerido como un marcador sensible de la función renal ${ }^{16-22}$; sin embargo, estudios recientes han demostrado que al igual que la creatinina, la cistatina $\mathrm{C}$ se ve afectada por la edad, el sexo, la masa muscular y la enfermedad del hígado y sobreestima la función renal en pacientes con cirrosis $^{17-29}$. En conclusión la medición de Crs deben utilizarse para evaluar la función renal en pacientes con cirrosis avanzada, hasta que métodos más fiables de medición de la función renal se generalicen ${ }^{29}$.

EL SHR se define como la insuficiencia renal que ocurre en pacientes con enfermedad hepática, ya sea aguda o crónica, con hipertensión portal, en ausencia de evidencia clínica de laboratorio y anatómica de otras causas conocidas de falla renal.

La incidencia anual del SHR en pacientes con cirrosis y ascitis ha sido estimada en $8 \%$. La probabilidad anual de desarrollar SHR en pacientes cirróticos es estimada en $18 \%$ a un año y en $39 \%$ a 5 años. Debido a la naturaleza funcional de la falla renal no hay marcador específico de SHR ${ }^{11,20}$. El SHR es la complicación asociada a cirrosis con peor pronóstico y es considerado como el evento terminal de la enfermedad.

Aunque el SHR es la causa más común de azoemia en pacientes con cirrosis avanzada, otras causas de falla renal deben descartarse en estos pacientes. Por 
lo tanto, el primer paso en el manejo de pacientes cirróticos con deterioro de su función renal u oliguria es un correcto diagnóstico de la etiología del deterioro renal.

Como desafortunadamente no hay prueba específica para hacer un diagnóstico conclusivo del SHR, su adecuado diagnóstico está basado sobre la exclusión de otros tipos de falla renal que puedan ocurrir en este grupo de pacientes, por lo tanto el SHR, es un

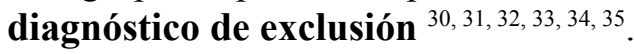

Se deben tener en cuenta 2 aspectos básicos a la hora de hacer ese diagnóstico: el primero, es la reducción de la rata de filtración glomerular y el segundo, es diferenciar el SHR de otras causas de insuficiencia renal.

En cuanto al primer punto, se debe tener en cuenta que la masa muscular y, por lo tanto, la liberación de creatinina está considerablemente reducida en este grupo de pacientes, por lo cual los pacientes cirróticos pueden tener creatinina en rango normal con un rata de filtración glomerular marcadamente disminuida; igualmente la urea, la cual es sintetizada por el hígado, se encuentra reducida como consecuencia de la insuficiencia hepática (tabla 1).

Debido a la falta de especificidad de marcadores para el SHR, en 1996 fueron publicados los primeros criterios diagnósticos del SHR, propuestos por el Club Internacional de la Ascitis y que fueron basados sobre los 3 principales conceptos de la época:

1. La insuficiencia renal en el SHR es funcional y causada por una severa vasoconstricción arteriolar renal.

2. El SHR ocurre en pacientes con disfunción circulatoria sistémica causada por una vasodilatación extrarrenal.

3. La expansión del volumen no mejora la función renal.

Sin embargo, debido al mejor entendimiento de la patogénesis del SHR y a la introducción de nuevas herramientas terapéuticas, surgió la necesidad de replantear su definición y establecer nuevos criterios por el Club Internacional de la Ascitis en el año 2007.

Las principales diferencias con los criterios de 1996 son:
1. La potencial reversibilidad del SHR aun sin trasplante hepático.

2. El rol dominante de la vasodilatación arterial en el árbol esplénico.

3. El rol frecuente de la peritonitis bacteriana espontánea como factor precipitante en el SHR tipo 1.

Los criterios establecidos por el Club Internacional de la Ascitis 2007 para el SHR son:

Las principales diferencias entre los 2 criterios diagnósticos (antiguos y nuevos) son:

a. Exclusión del aclaramiento de creatinina de 24 horas, debido a que este es más complicado que una Crs para propósitos de rutina y no incrementa la exactitud en cuanto a estimar la función renal en pacientes cirróticos.

\section{Tabla 1}

Criterios diagnósticos del síndrome hepatorrenal propuestos por el Club Internacional de la Ascitis, 1994

Criterios mayores:

1. Presencia de enfermedad hepática aguda o crónica con falla hepática e hipertensión portal.

2. Ausencia de shock, depleción de volumen, proceso infeccioso en curso, fármacos nefrotóxicos.

3. Disminución de la rata de filtración manifestada por una Crs mayor de $1.5 \mathrm{mg} / \mathrm{dl}$ o un aclaramiento de creatinina de 24 horas menor de $40 \mathrm{ml} / \mathrm{min}$.

4. No mejoría de la función renal (disminución de la Crs a valores menores de $1.5 \mathrm{mg} / \mathrm{dl}$ o un incremento en el aclaramiento de la creatinina en 24 horas mayor de $40 \mathrm{ml} / \mathrm{min}$ ) después de la suspensión de los diuréticos y de la expansión del volumen plasmático con 1.5 1. de expansores del volumen plasmático.

5. Proteinuria menor de $500 \mathrm{mg}$ /día y ausencia de uropatía obstructiva o enfermedad renal parenquimatosa por ecografía.

\section{Criterios menores:}

1. Volumen urinario $<500 \mathrm{ml} /$ día.

2. Sodio urinario $<10 \mathrm{meq} / 1$.

3. Osmolalidad urinaria mayor que la plasmática.

4. Glóbulos rojos en orina menores de 50 por campo de alto poder.

5. Concentración sérica de sodio menor de $130 \mathrm{meq} / 1$.

Fuente: Arroyo V, Ginés P, Gerbes A, et al. Definition and diagnostic criteria of refractory ascites and hepatorrenal syndrome in cirrhosis. Hepatology 1996;23:164-176 $6^{35}$. 
b. La falla renal funcional se puede presentar en el sitio de un proceso infeccioso bacteriano, pero en ausencia de choque séptico. Esto significa que el tratamiento del SHR puede ser iniciado sin esperar que se complete la recuperación de la función renal.

c. La expansión de volumen debe ser con albúmina más que con solución salina, debido a que la primera causa una mejor y más sostenida expansión.

d. Los criterios menores fueron excluidos ya que el panel de expertos concluyó que no son esenciales.

Recientemente la octava conferencia de consenso internacional del Grupo de Iniciativa para la Calidad de Diálisis Aguda ${ }^{37-46}$ (ADQI, por sus siglas en inglés) propuso lo que denominó desórdenes o disfunciones renales asociados a cirrosis. El consenso final del grupo de trabajo propuso aplicar los criterios RIFLE para definir AKI en pacientes con cirrosis, independientemente de si la causa del deterioro agudo de la función renal estaba relacionado con un trastorno funcional o estructural ${ }^{47}$. (Tabla 2) (Tabla 3).

\section{Tabla 2}

Criterios establecidos por el Club Internacional de la Ascitis 2007 para el SHR

1. Cirrosis con ascitis.

2. $\mathrm{Crs}>1.5 \mathrm{mg} / \mathrm{dl}(>133 \mathrm{umol} / \mathrm{l})$.

3. No mejoría de la Crs (disminución a un nivel $<133$ umol/1) después de 2 días de la suspensión del diurético y de la expansión de volumen con albúmina. La dosis recomendada de albúmina es de $1 \mathrm{gr} / \mathrm{kg} /$ día, hasta un máximo de $100 \mathrm{gr} /$ día.

4. Ausencia de shock.

5. Ausencia actual o reciente de drogas nefrotóxicas.

6. Ausencia de enfermedad parenquimatosa renal sugerida por la presencia de proteinuria $>500 \mathrm{mg} /$ día, hematuria $(>50$ células rojas por campo de alto poder) o anormalidad renal por ecografía ${ }^{36}$.

Fuente: Gut 2007;56;1310-1318.

\section{Clínica del síndrome hepatorrenal}

Cuando se está frente a un paciente con enfermedad hepática y que presenta deterioro de su función renal se debe pensar en 3 posibilidades, que son las más frecuentes en este tipo de pacientes: SHR, azoemia prerrenal y necrosis tubular aguda, sin querer decir con esto que no se deba pensar en otras causas de falla renal que son menos frecuentes ${ }^{15}$.

\section{Diagnóstico diferencial de insuficiencia renal aguda en pacientes cirróticos}

Se conocen clínicamente 2 tipos de $\mathrm{SHR}^{7}$ :

El SHR tipo 1, es caracterizado por una severa y rápida falla renal progresiva, definida por el doblaje de la Crs, alcanzando un nivel mayor de $2.5 \mathrm{mg} / \mathrm{dl}$ en menos de 2 semanas. El SHR tipo 1 puede aparecer espontáneamente durante el curso de la enfermedad, pero lo más común es que se relacione con un evento precipitante, siendo el más frecuente un proceso infeccioso tipo peritonitis bacteriana espontánea (PBE) seguido por hemorragia de vías digestivas, un procedimiento quirúrgico mayor o una hepatitis aguda sobreimpuesta sobre una cirrosis. Los pacientes que presentan PBE, desarrollan el SHR en un $25 \%$. Sin tratamiento el SHR tipo 1 implica un pésimo pronóstico y una supervivencia de aproximadamente 2 semanas después del inicio de la falla renal.

\begin{tabular}{|c|c|c|}
\hline \multicolumn{3}{|c|}{ Tabla 3} \\
\hline \multicolumn{3}{|c|}{$\begin{array}{l}\text { La iniciativa para la calidad de diálisis aguda } \\
\text { (ADQI) los criterios para la definición y } \\
\text { clasificación de la lesión renal aguda } \\
\text { (modificados criterios RIFLE) })^{37}\end{array}$} \\
\hline $\begin{array}{l}\text { AKI } \\
\text { Etapa }\end{array}$ & $\begin{array}{c}\text { Criterios de } \\
\text { creatinina sérica }\end{array}$ & $\begin{array}{c}\text { Criterios de salida } \\
\text { de la orina }\end{array}$ \\
\hline $\begin{array}{c}1 \\
\text { (Riesgo) }\end{array}$ & $\begin{array}{l}\text { Aumentar Scr } \geq 0,3 \mathrm{mg} / \mathrm{dl} \\
\text { dentro de las } 48 \text { horas o un } \\
\text { aumento del } 150-200 \% \text { (de } \\
1,5 \text { a } 2 \text { veces) de la línea } \\
\text { de base }\end{array}$ & $\begin{array}{l}<0,5 \mathrm{ml} / \mathrm{kg} / \text { hora } \\
\text { durante }>6 \text { horas }\end{array}$ \\
\hline $\begin{array}{c}2 \\
\text { (lesión) }\end{array}$ & $\begin{array}{l}\text { Aumentar Scr } 200 \% \text { a } 299 \% \\
\text { ( } \geq 2 \text { a } 3 \text { veces) desde el } \\
\text { inicio }\end{array}$ & $\begin{array}{l}<0,5 \mathrm{ml} / \mathrm{kg} / \text { hora } \\
\text { durante }>12 \text { horas }\end{array}$ \\
\hline $\begin{array}{c}3 \\
(\text { FALLA) }\end{array}$ & $\begin{array}{l}\text { Aumentar Scr } \geq 300 \% \text { ( } \geq 3 \\
\text { veces) desde el inicio o Scr } \\
\geq 4,0 \mathrm{mg} / \mathrm{dl} \text { con un aumen- } \\
\text { to agudo de } \geq 0,5 \mathrm{mg} / \mathrm{dl} \mathrm{o} \\
\text { iniciación de la terapia de } \\
\text { reemplazo renal }\end{array}$ & $\begin{array}{l}<0,3 \mathrm{ml} / \mathrm{kg} \text { /hora } \\
\text { durante } 24 \text { horas o } \\
\text { anuria durante } 12 \\
\text { horas }\end{array}$ \\
\hline
\end{tabular}

$\mathrm{SCR}=$ creatinina sérica 


\begin{tabular}{|l|c|}
\hline \multicolumn{2}{|c|}{ Tabla 4} \\
\hline \multicolumn{2}{|c|}{$\begin{array}{c}\text { Criterios diagnósticos propuestos para } \\
\text { la disfunción renal en la cirrosis } 47\end{array}$} \\
\hline Diagnóstico & \multicolumn{1}{c|}{ Definición } \\
\hline \multirow{2}{*}{$\begin{array}{l}\text { Lesión } \\
\text { renal aguda }\end{array}$} & $\begin{array}{l}\text { Un aumento de Scr } \geq 50 \% \text { del valor inicial o } \\
\text { un aumento Scr }>0,3 \mathrm{mg} / \mathrm{dl}\end{array}$ \\
\cline { 2 - 3 } & $\begin{array}{l}\text { Tipo-1 SHR es una forma específica de la } \\
\text { lesión renal aguda }\end{array}$ \\
\hline $\begin{array}{l}\text { Enfermedad } \\
\text { renal crónica }\end{array}$ & $\begin{array}{c}\text { - TFG }<60 \mathrm{ml} / \mathrm{min} \text { durante }>3 \text { meses calculado } \\
\text { según MDRD-6 fórmula }\end{array}$ \\
\hline \multirow{2}{*}{$\begin{array}{l}\text { IR aguda en } \\
\text { la enfermedad } \\
\text { renal crónica }\end{array}$} & $\begin{array}{l}\text { Aumento de Scr } \geq 50 \% \text { del valor basal o un } \\
\text { aumento de Scr }>0,3 \mathrm{mg} / \mathrm{dl} \text { en un pacien- } \\
\text { te con cirrosis que la TFG es }<60 \mathrm{ml} / \mathrm{min} \\
\text { durante }>3 \mathrm{meses} \text { calculado según MDRD-6 } \\
\text { fórmula }\end{array}$ \\
\hline
\end{tabular}

TFG, la tasa de filtración glomerular; SHR, síndrome hepatorrenal; Scr, creatinina sérica. Tanto el deterioro agudo de la función renal y el fondo la disfunción renal crónica pueden ser funcionales o estructurales en la naturaleza. MDRD-6: TFG $=170 \times \mathrm{Scr}(\mathrm{mg} / \mathrm{dL})$ $-0.999 \times$ edad $-0.176 \times 1.180$ (si es negro) $\times 0.762$ (si es mujer $) \times$ suero urea nitrógeno $-0,170 \times$ albúmina 0.138 . (Tabla 4 )

\section{El SHR tipo 2:}

Se caracteriza por una insuficiencia renal que no se establece en forma rápida y una reducción de la función renal (Crs de 1.5 a $2.5 \mathrm{mg} / \mathrm{dl}$ ) que ocurre durante semanas o meses. Puede también aparecer espontáneamente o por un evento precipitante, pero por lo general es asociado con ascitis refractaria. $\mathrm{La}$ supervivencia de este grupo de pacientes es mejor ( 4 - 6 meses) que la de los pacientes con SHR tipo 1 , pero peor que la de pacientes cirróticos no azoémicos con ascitis (Tabla 5).

\section{Tratamiento}

Aunque el SHR es la causa más común de azoemia en pacientes con cirrosis avanzada, otros tipos de falla renal deben descartarse. Por lo tanto, el primer paso en el manejo de pacientes con enfermedad hepática aguda o crónica con falla renal es el correcto diagnóstico de la etiología del deterioro de su función renal ${ }^{8}$.

\section{Prevención del SHR}

Si se acepta que existen situaciones conocidas que pueden precipitar el SHR como son: infecciones, en

\begin{tabular}{|l|c|c|c|}
\hline \multicolumn{4}{|c|}{ Tabla 5 } \\
\hline \multicolumn{3}{|c|}{ Diagnóstico diferencial de insuficiencia renal } \\
aguda en pacientes cirróticos \\
\hline & SHR & Prerrenal & Nec tub aguda \\
\hline Sedi urinario & Normal & Cilin hialinos & $\begin{array}{c}\text { Cilin granulosos/ } \\
\text { células epiteliales }\end{array}$ \\
\hline FENA & $<1$ & $<1$ & $>2$ \\
\hline NA urinario & $<10$ & $<20$ & $>40$ \\
\hline $\begin{array}{l}\text { OSM } \\
\text { urinaria }\end{array}$ & Variable & $>500$ & $<300$ \\
\hline $\begin{array}{l}\text { Dens } \\
\text { específica }\end{array}$ & $>1.2$ & $>1.20$ & $\sim 1.010$ \\
\hline $\begin{array}{l}\text { Relación } \\
\text { Creat U/P }\end{array}$ & $>40: 1$ & $>40: 1$ & $<20: 1$ \\
\hline $\begin{array}{l}\text { Resp a } \\
\text { expansores } \\
\text { plasmáticos }\end{array}$ & Mala & Buena & Variable \\
\hline
\end{tabular}

especial la peritonitis bacteriana espontánea, hipovolemia inducida por sangrado digestivo o las paracentesis de grandes volúmenes sin reposición de albúmina; la prevención debe partir del adecuado conocimiento y tratamiento de estas situaciones.

Se han publicado estudios controlados randomizados en los cuales el SHR se puede prevenir en situaciones clínicas especiales:

1. La incidencia de SHR en pacientes con PBS puede ser reducida por la administración de albúmina. Al momento del diagnóstico de PBS, además del tratamiento antibiótico, se recomienda albúmina con una dosis inicial, el primer día de $1.5 \mathrm{gr} / \mathrm{kg}$ de peso corporal y de $1 \mathrm{gr} / \mathrm{kg}$ al día tercero, hasta una dosis máxima de 100 y 150 gr, respectivamente. La infusión de albúmina ha demostrado reducir la incidencia del SHR tipo 1 y reducción de mortalidad (10\% de incidencia de SHR tipo 1 en el grupo que recibe albúmina vs. 33\% en el grupo control y mortalidad de $22 \%$ grupo albúmina vs. $41 \%$ grupo control)12.

2. Previniendo la aparición de PBS. La profilaxis primaria de PBS en pacientes de alto riesgo, usando quinolonas es asociada con una disminución significativa de desarrollo de PBS y de SHR tipo 
1, como de también de un incremento de la supervivencia de 3 y 12 meses.

Por lo tanto, se recomienda la profilaxis en pacientes cirróticos con ascitis con sangrado gastrointestinal; la administración por corto tiempo (7 días) de norfloxacina $400 \mathrm{mg}$ cada 12 horas es sugerida. El uso de antibiótico por largo tiempo (norfloxacina oral $400 \mathrm{mg} /$ día) es recomendado en pacientes que han tenido episodios previos de $\mathrm{PBS}^{48-57}$.

\section{Opciones de tratamiento en SHR:}

\section{Tratamiento farmacológico:}

El tratamiento con vasoconstrictores y albúmina es el tratamiento de elección para el SHR tipo 134. El objetivo del tratamiento es producir una vasoconstricción en el lecho vascular esplénico así como reducir la hipovolemia efectiva.

El estudio de la fisiopatología del SHR y basados, sobre todo, en la vasodilatación arterial del árbol esplénico, han llevado a los diferentes investigadores al estudio y uso de fármacos vasoconstrictores de la circulación esplénica, con el objeto de revertir farmacológicamente y en una forma más fisiológica la disfunción circulatoria del SHR y así mejorar la función renal.

La primera droga usada para este propósito fue la dopamina, sin embargo, posteriores estudios demostraron que la administración de dopamina en pacientes cirróticos con ascitis, con y sin SHR tiene poco efecto sobre la función renal. Desde entonces, numerosos vasoconstrictores han sido estudiados.

Hasta la fecha se han utilizado 3 tipos de agentes vasoconstrictores en el tratamiento del $\mathrm{SHR}^{56}$ :

a. Análogos de la somatostatina: octreótido

b. Análogos de la vasopresina: ornipresina, terlipresina

c. Agonistas alfa adrenérgicos: norepinefrina, midrodine

La ornipresina y la terlipresina son los análogos de la vasopresina que se han usado en el SHR, sin embargo, la ornipresina a pesar del beneficio demostrado en la reversión del SHR ha sido abandonada por sus efectos isquémicos: arritmias cardiacas, isquemia miocárdica, necrosis cutánea.

La terlipresina o triglicil - lisina - vasopresina, es un derivado sintético de la vasopresina que posee una acción dominante sobre receptores V1, lo que explica su potente efecto vasoconstrictor; su vida media plasmática es más larga (4-10 horas) que la de otros análogos, lo que facilita su administración en bolos intravenosos en vez de infusión continua. Hasta la fecha es el vasoconstrictor más ampliamente estudiado, usado en el SHR tipo 1 y disponible en nuestro país.

La dosis inicial es de $1 \mathrm{mg}$ cada $4-6$ horas. Si no hay respuesta (disminución de la Crs en un 25\% después de 2 días), la dosis puede ser doblada cada 2 días hasta un máximo de $12 \mathrm{mg} /$ día. El tratamiento puede ser interrumpido si la Crs no disminuye en un $50 \%$ después de 7 días de estar con la dosis máxima o si no hay una reducción después de 3 días. En pacientes con respuesta temprana, el tratamiento debe continuarse hasta la reversión del SHR o por un máximo de 14 días o por la presencia de efectos secundarios isquémicos y arritmias inducidas por la terlipresina.

La administración concomitante de expansores de volumen tipo albúmina puede mejorar el efecto de los vasoconstrictores. La dosis recomendada de albúmina es de $1 \mathrm{gr} / \mathrm{kg}$ de peso corporal como dosis inicial, hasta un máximo de 100 gr y continuar con 20 - 40 gr/día (Tabla 6).

Con el uso de terlipresina y albúmina, hasta el 60\% de los SHR se pueden revertir. La insuficiencia renal puede recurrir hasta en un $15 \%$ después de la descontinuación del tratamiento, pero el reinicio del tratamiento es igualmente efectivo.

Una alternativa muy disponible en nuestras instituciones es la norepinefrina a una dosis de 0.5 -3 mg IV/hora en infusión continua, hasta lograr un incremento de la presión arterial de $10 \mathrm{mmHG}$.

El midrodine, un fármaco de uso oral, ha sido usado en pacientes con SHR tipo 1, a una dosis de $7.5 \mathrm{mg}$ (máxima $12.5 \mathrm{mg}$ ), cada 8 horas, más octreótido 100 


\begin{tabular}{|c|l|}
\multicolumn{2}{|c|}{ Tabla 6 } \\
\multicolumn{2}{|c|}{ Fármacos vasoconstrictores para el tratamiento } \\
del síndrome hepatorrenal
\end{tabular}

MAP: presión arterial media

Fuente: Nadim et al. Critical Care 2012 16: R23 doi: 10.1186 / cc11188

mg subcutáneo, cada 8 horas, asociado a expansión de volumen con albúmina en una dosis de $20-40$ gr IV/día ${ }^{23}$.

El octreótido un octapéptido análogo de la somatostatina con potente acción vasoconstrictora sobre la vasculatura esplénica, ha sido usado en el SHR, a una dosis de 100 ug SC cada 8 horas, hasta una dosis máxima de 200 ug SC cada 8 horas $^{23,26}$.

Hasta la fecha no hay estudios que soporten el uso de vasoconstrictores en el SHR tipo 2.

\section{Derivaciones (shunt) portosistémicas transyugu- lar intrahepático (TIPS)}

El desarrollo de la derivación intrahepática transyugular portocava, (TIPS de la literatura anglosajona: Transyugular intrahepatic portocaval shunt) obvia la necesidad de realizar un procedimiento quirúrgico mayor como es la derivación portocava (DPV), sin embargo, la inserción de un TIPS no es un simple procedimiento y no está exenta de complicaciones. Desafortunadamente, no hay estudios adecuadamente controlados para valorar su eficacia en el SHR.

Los TIPS funcionan como una derivación portocava por lo que se espera que mejoren la hipertensión portal. Su inserción es asociada con un incremento del gasto cardiaco y una expansión del flujo sanguíneo central. El efecto simultáneo sobre la circulación esplénica y sistémica puede representar el mecanismo por el cual los TIPS mejoran la perfusión renal, la filtración glomerular y la excreción de sodio urinario y agua.

En términos generales los TIPS en los pequeños estudios han demostrado que mejoran la función renal y eliminan la ascitis. En los pacientes con SHR tipo 1 puede mejorar la supervivencia, algo que no se ha demostrado en el SHR tipo 2. No debería ser usado en pacientes con bilirrubina $>5 \mathrm{mg} / \mathrm{dl}$, infección bacteriana, presencia de encefalopatía hepática o historia de encefalopatía recurrente, disfunción cardiaca o pulmonar severa, o un score Child-Pugh $>11^{31}$.

\section{Diálisis renal o hepática}

La terapia de reemplazo renal ha sido usada en el manejo de los pacientes con SHR. Se han usado hemodiálisis (HD) intermitente, hemodiafiltración venovenosa (HDVV), hemofiltración de alto volumen y diálisis peritoneal (DP), aunque no hay pruebas controladas que evalúen su efectividad en esta situación ${ }^{59-67}$.

La hipotensión sistémica hace que, frecuentemente, la HD no sea factible en este grupo de pacientes, lo mismo que la presencia de ascitis y peritonitis reduce la eficiencia de la DP. Sin embargo, la terapia de reemplazo renal es usada en muchos centros como una terapia puente mientras sale el trasplante hepático $^{64}$.

Debido a que la mayor causa de muerte en falla hepática y en cirrosis descompensada es el edema cerebral, resultado del aumento de la presión intracraneana y de la disminución de la presión de perfusión cerebral, la HDVVC o hemofiltración venenosa continua (HVVC) han sido recomendadas como terapia de reemplazo renal en este tipo de pacientes. 
La naturaleza continua del procedimiento junto con la falta de cambios bruscos en la presión arterial media y en la presión intracraneana, permite una mejor remoción de toxinas urémicas así como mediadores de inflamación y una mejor estabilidad cardiovascular $^{65}$.

Varios métodos de soporte hepático extracorpóreo o diálisis hepática están siendo usados en pacientes con falla hepática aguda o crónica agudizada, como es el caso del SHR, con incrementada frecuencia como terapia puente mientras sale el trasplante hepático. El objetivo de los sistemas bioartificiales sería, en teoría, reemplazar completamente la función hepática tanto en desintoxicación como en síntesis, sin embargo, la complejidad de ambas funciones para llevarla a cabo por los diferentes sistemas utilizados sigue siendo un reto. Por lo tanto, utilizar máquinas que solo cumplan la función de desintoxicación conocidas como diálisis hepática, las cuales remueven de la circulación toxinas solubles unidas a la albúmina. El sistema recirculante absorbente molecular (MARS) es una variante de diálisis con albúmina desarrollada por Gambro e introducida desde $1999^{62}$. Prometheus, es otra opción de diálisis hepática basada en la separación fraccionada del plasma y desarrollada por Fresenius. Aunque los datos preliminares de pequeños estudios, soporten seguridad y eficacia con ambos tipos de terapia, ambos métodos deben considerarse experimentales hasta que grandes pruebas controladas y randomizadas demuestren beneficio de supervivencia ${ }^{65}$.

\section{Trasplante hepático:}

Es el tratamiento de elección tanto en el SHR tipo 1 como el 2. Sin embargo, el principal problema del trasplante hepático (TH) en el SHR tipo 1, es su aplicabilidad. Desafortunadamente, la pobre supervivencia de los pacientes con SHR, sobre todo del tipo 1 (días a semanas), la escasa disponibilidad de órganos y las diferentes barreras administrativas de nuestro Sistema de Salud hacen la aplicabilidad del trasplante hepático muy improbable en este tipo de pacientes ${ }^{40}$.

E1 TH es considerado ser el tratamiento ideal para los pacientes con enfermedad hepática terminal ${ }^{40-42}$.
Muchos de estos pacientes son admitidos por varios grados de disfunción renal concomitante, incluyendo SHR. El TH puede revertir el SHR. Los pacientes con SHR que se someten a TH tienen más complicaciones, estancia hospitalaria en UCI más prolongada y mayor mortalidad intrahospitalaria que los pacientes trasplantados sin SHR. Igualmente, la supervivencia a largo tiempo de los pacientes trasplantados con SHR es buena, de $60 \%$ a 3 años, levemente menor a la de los pacientes trasplantados sin SHR (70 $-80 \%$ a 3 años) $)^{41}$.

Las anormalidades hemodinámica y neurohormonal asociadas a SHR desaparecen al mes después de la cirugía y los pacientes recuperan la habilidad para excretar el sodio y el agua.

Con un agresivo manejo pre y postrasplante se puede conseguir un excelente resultado en pacientes con SHR con el TH. En este sentido la búsqueda de métodos terapéuticos diferentes al TH para mejorar la función renal o hepática, ya sea en forma temporal o permanente (incluso para revertir el SHR), o como terapia puente hasta que aparezca el trasplante hepático, puede ser beneficiosa ${ }^{40}$.

\section{Recomendaciones finales para el tratamiento:}

SHR tipo 1:

El uso de vasoconstrictores combinados con albúmina se considera la terapia de primera línea, en caso de ausencia de respuesta se puede intentar el uso de TIPS. Los tratamientos de reemplazo, ya sea hepático o renal, también se pueden usar como terapia puente mientras sale el trasplante hepático.

En el SHR tipo 2 no existe soporte para el uso de vasoconstrictores. Los TIPS pueden ser usados para mejorar la ascitis refractaria ${ }^{67}$.

\section{Agradecimiento:}

Al Dr. Gustavo Aroca por su apoyo y colaboración .

\section{Conflicto de interés}

El autor declara que no tiene conflictos de interés relacionados con la publicación o con los contenidos de este artículo. 


\section{Bibliografía}

1. Helwing FC, Scuhtz CB. A liver-Kidney sindrome. Clinical, pathological and experimental studies. Surg Gynecol Obst 1.932;55:570-580.

2. Wilensky AO. Occurrence, distribution and pathogenesis of so - called liver death and / or hepatorenal syndrome. Arch Surg 1939;38:625-631.

3. Epstein M. Effects of heart and liver disease and neoplasia on kidney and electrolyte metabolism. In: Massry \& Glassok's. Textbook of nephrology. 4st ed. Baltimore: Williams\& Wilkins, 2000:1067-1077.

4. Flint A. Clinical report on hydro- peritoneum based on a analysis of forty -six cases. Am J med Sci 1863;45:306-339.

5. Hecker R, Sherlock S, Electrolyte and circulatory changes in terminal liver failure. Lancet 1956;2:1221-1225.

6. Koppel MH, Coburn JN et al. Transplantation of cadaveric kidneys from patients with hepatorenal syndrome. Evidence for the functional nature of renal failure in advanced liver disease. N Engl J Med 1969;280:1367-1371.

7. Iwatsuki S, Popovtzer MM, et al. Recovery from "hepatorenal syndrome" after orthotopic liver transplantation. N Engl J med 1973;1973;289:1155-1159.

8. Paper S: Hepatorenal syndrome. In Epstein M (ed): The Kidney in Liver Disease, 2 nd ed. New York, Elsevier, 1983, p 87.

9. Shear L, Kleinerman J, Gabuzda GJ: Renal failure in patients with cirrhosis of the liver. I. Clinical and pathologic characteristics. Am J med 39:184,1965.

10. Bataller R, Gines P, Guevara M, Arroyo V. Hepatorenal syndrome. Semin Liver Dis 1997;17:233-248.

11. Bernardo DE, Summerskill WH, Strong CG, Baldus WP. Renal function, rennin activity and endogenous vasoactive substances in cirrhosis. Am J Dig Dis 1970;15:419-425.

12. Dibona GF. Renal nerve activity in hepatorenal syndrome. Kidney Int 1984;25:841-853.

13. Cupin W. Diagnosis and pathophysiology of hepatorenal syndrome. Available from: URL: http//:internet links (http:// www.hden.com/symp/02asnb/kup/kup.htm.

14. Bolton C, Barnard WG. The pathological occurrence of the liver in experimental venous stagnation. J Pathol Bacteriol 1931;34:701-706.

15. Papper S. The role of the kidney in Laennec's cirrhosis of the liver. Medicine 1958;37:299-316.

16. Perera GA. The plasma volume in Laennec's cirrhosis of the liver. Ann Intern Med 1946;24:643-648.

17. Lieberman FL, Denison EK, Reynolds TB. The relationship of plasma volume, hypertension portal, ascites and renal sodium, and water retention in cirrhosis: the "overflow" theory of ascitis formation. Ann N Y Acad Sci 1970;170:202-206.

18. Schier RW, Arroyo V Bernardi M, et al. Peripheral arterial vasodilation hypothesis: a proposal for the initiation of renal sodium and water retention in cirrhosis. Hepatology 1988;8:1151-1157.

19. Ring-Larsen H . Renal blood flow in patients with hepatorenal syndrome. Scand J Clin Invest. 1977 Nov;37(7):635-42.

20. Asbert M et al. Humoral response to cirrhosis .Gastroenterology .1993 May; 104(5):1485-91.

21. Ginés P, Fernández-Esparrach G, Arroyo V, Rodés J .Pathogenesis of ascitis in cirrhosis. Semin Liver Dis 1997;17:175-90.

22. Arroyo V, Ginés P, Jiménez W, Rodés J. Ascitis, renal failure and electrolyte disorders in cirrhosis. Pathogenesis, diagnosis and treatment. In : McIntyre N, Benhamou JP, Bircher J,et al, eds. Oxford: Oxford medical publications, 1991:429-470.

23. Laffi G, La Villa G, Pinzani M, et al. Arachidonic acid derivatives and renal function in liver cirrhosis. Semin Nephrol 1997; 17:530-548.

24. Ros J, Jiménez W, Bosch-Marcé M, et al. Role of nitric oxide and prostacyclin in the control of renal perfusion in experimental cirrhosis. Hepatology 1995;21:915-920.

25. Quintero E, Ginés P, Arroyo V, et al. Sulindac reduces the urinary excretion of prostaglandins and impairs renal function in cirrhosis with ascitis. Nephron 1986;42:298-303. 
26. Ginés P, Schrier RW. Hepatorenal Syndrome and renal dysfunction associated with liver disease. In: Schrier RW, Gottschalk CW, eds. Diseases of the kidney. Boston: Little, Brown and Co, 1997:2099-2127.

27. Paper S Belsky JL, Bleifer KH. Renal failure in Laennec's cirrhosis of the liver. I. Description of clinical and laboratory features. Ann Intern Med 1959;51:759.

28. Epstein M: The Hepatorenal Syndrome. Emerging perspective of pathophysiology and therapy. J Am Soc Nephrol 1994;4:173594.

29. Epstein M, Oster JR, DeVelasco RE: Hepatorenal syndrome following hemihepatectomy. Clin Nephrology 1976;5:128.

30. Arroyo V, Ginés P, Gerbes A, et al. Definition and criteria diagnostic of refractory ascites and hepatorenal syndrome in cirrhosis. Hepatology 1996;23:164-176.

31. Arroyo V, Rodés J. A rational approach to the treatment of ascitis. Postgrad Med J 1975;51:558-562.

32. Ginés J, Schrier RW. Hepatorenal Syndrome and renal dysfunction associated with liver disease. In: Schrier RW, Gottschalk CW, eds. Diseases of the kidney. Boston: Little, Brown and Co, 1997:2099-2127.

33. Ignatius KP. Hepatorenal Syndrome. In: Johnson RJ, Feehally J, eds. Comprehensive Clinical Nephrology. 1ed. Barcelona: Mosby; 2000,4:17.1-17.8.

34. Takabatake $\mathrm{T}$ et al. Discrepancy between creatinine clearance and rate filtration glomerular in patients with liver diseases. Arch Inter Med 1988 Jun;148(6):1313-1315.

35. Ginés P, Rodés J. Clinical disorders of renal function in cirrhosis with ascites. In: Arroyo V, Ginés P, Rodés J, Schrier RW. Ed. Ascites and renal dysfunction in liver disease $1^{\text {st }}$ ed. Massachusetts: Blackwell Science, Inc; 1999:36-62.

36. Horio M, Orita Y, Fukunaga M. Assessment of renal Function. In: Johnson RJ, Feehaly J, eds. Comprehensive Clinical Nephrology. $1^{\text {st }}$ ed. Barcelona: Mosby; 2000;2:3.1-4.10.

37. Nadim MK, Kellum JA, Davenport A, Wong F, Davis C, Pannu N, Tolwani A, Bellomo R, Genyk YS; ADQI Workgroup. Hepatorenal syndrome: the 8th International Consensus Conference of the Acute Dialysis Quality Initiative (ADQI) Group.Crit Care. 2012 Feb 9;16(1):R23.

38. Demirtas S, Bozbas A, Akbay A, et al. Diagnostic value of serum cystatina for evaluation of hepatorenal syndrome. Clin Chim Act .2001;311(2):81-89.

39. Eknoyan G. Glomerular abnormalities in liver disease. In: Epstein M, ed. The Kidney in liver disease. Baltimore: Williams\& Wilkins. 1988:154-181.

40. Platt JF, Ellis JH, Rubin Jm, et al. Renal duplex doppler ultrasonography: a noninvasive predictor of kidney dysfunction and hepatorenal failure in liver disease. Hepatology 1994;20:362-369.

41. Gonwa TA, Morris CA, Goldstein RM, et al.: Long-term survival and function renal following liver transplantation in patients with and without hepatorenal syndrome- Experience in 300 patients. Transplantation. 1991;51:428.

42. Iwatsuki S, Popovtzer MM, Corman JL, et al: Recovery from hepatorenal syndrome after orthotopic liver transplantation. N Eng J Med 289:1155,1973.

43. Wood RP, Ellis D, Starzl TE. The reversal of the hepatorenal syndrome in four pediatric patients following successful orthotopic liver transplantation. Ann Surg 205:415,1987.

44. Ortega R, Calahorra B, Ginés P. Vasoconstrictores en el tratamiento del Síndrome Hepatorrenal. Nefrología 2002 .Vol XXII;S 5:56-61.

45. Wong F, Blendis L: New challenge of hepatorenal syndrome: prevention and treatment. Hepatology. 2001; 34:1242-1251.

46. Ginés A, Ginés P, Escorcell A, Arroyo V et al. Incidence, predictive factors and prognosis of hepatorenal syndrome in cirrhosis. Gastroenterology 1993;105:229-236.

47. Barnardo DE, Baldus WP, Maher FT. Effects of dopamine on renal function in patients with cirrhosis. Gastroenterology 1970;58:524-531. 
48. Back Y, Gaudin C, Hadengue A, et al. Systemic, splanchnic and renal hemodynamic effects of dopaminergic dose dopamine in patients with cirrhosis. Hepatology 1991;14:483-487.

49. Wilson JR. Dopamine in hepatorenal syndrome. JAMA 1997;238:2719-2710.

50. Moore K. The Hepatorenal syndrome. Clinic Sci. 1997;92:433-443.

51. Angeli P, Volpin R, Gerunda G, et al. Reversal of type 1 hepatorenal syndrome with administration of midrodine and octroetido. Hepatolgy 1999;29:1690-1697.

52. Sabat M, Villanueva C, Rosello J et al. Effect of subcutaneous administration of octreotido on endogenous vasoactive system and renal function in cirrhotic patient with ascites. Dig Dis Sci 1998;43:2184-2189.

53. Lenz K, Hortnagl H, Druml W, et al. Ornipressin in the treatment of functional renal failure in decompensated liver cirrhosis. Gastroenterology 1991;101:1060-1067.

54. Gulberg V, Bilzer M, Gerbes AL. Long-term therapy and retreatment of hepatorenal syndrome type 1 with ornipressin and dopamine. Hepatology 1999;30:870-875.

55. Escorsell A, Bandi JC, Moitinho E, Rodés J, et al. Time profile of the hemodynamic effects of terlipressin in portal hypertension. J Hepatol 1997;26:621-627.

56. Ganne-Carrie N, Hadengue A, Benjamou JP. Hepatorenal syndrome. Long-term treatment with terlipressin as a bridge to liver transplantation. Dig Dis Sci. 1996;41:1054-1056.

57. Uriz J, Ginés P, Sort P, Jiménez W, et al. Terlipressin plus albumin infusion: an effective and safe therapy of hepatorenal syndrome. J Hepatology 2000;33:43-48.

58. Mulkay JP, Louis H, Deviere J, et al. Long-term terlipresin administration improves renal function in cirrhotic patients with type 1 hepatorenal syndrome: pilot study. Acta gastroenterol Belg 2001;64:15-19.

59. Arroyo V, Bataller R, Guevara M. Treatment of hepatorenal syndrome in cirrhosis In: Arroyo V, Ginés P, Rodés J, Schrier RW eds. Ascites and renal dysfunction in liver disease $1^{\text {st }}$ ed. Massachusetts: Blackwell Science, Inc; 1999:492-510.

60. Epstein M. Hepatorenal syndrome. In: Wolfe M eds. Therapy of digestive disorders. A companion to Sleisenger and Fortrans Gastrointestinal and liver disease, $1^{\text {st }}$ ed .Philadelphia, Saunders Company, 2000:398-404.

61. Ellis D, Avner ED, Renal failure and dialysis therapy in children with failure hepatic in the perioperative period of orthotopic liver transplantation. Clin Nephrol 1986;25:295-303.

62. Epstein M, Perez GO. Continuous arteriovenous ultrafiltration in the management of the renal complications of liver disease. Int J Artif Organs. 1985;9:215-216.

63. Davenport A, Will EJ, Davison AM. Effect of renal replacement therapy on patients with combined acute renal and fulminant hepatic failure. Kidney Int Suppl. 1993 Jun;41:S 245-51.

64. Improved cardiovascular stability during continuous modes of renal replacement therapy in critically ill patient with hepatic and renal failure. Crit Care Med. 1993 Mar;21(3):328-38.

65. Davenport A. The management of renal failure in patient at risk of cerebral edema/hypoxia. New Horiz. 1995 Nov; 3(4):717-24.

66. Iwai H, Naki M, Naito T, et al. Removal of endotoxin and cytokines by plasma exchange in patients with acute hepatic failure. Crit Care Med. 1998 May;26(5):873-6.

67. Bellomo R, Baldwin I, Ronco C. Preliminary experience with high-volume hemofiltration in human septic shock. Kidney Int Suppl. 1998 May;66:S182-5.

68. Wong F, Blendis L. New Challenge of hepatorenal syndrome: prevention and treatment. Hepatolgy 2001;34:1242-1251. 